\title{
Relaxation process features of photoconductivity in p-i-n structures
}

\author{
R.A. Mumimov ${ }^{1}$, Sh.K. Kanyazov², A.K. Saymbetov ${ }^{1}$ \\ ${ }^{I}$ Physical-Technical Institute, 100084 Tashkent, Uzbekistan \\ E-mail:detector@uzsci.net \\ ${ }^{2}$ Karakalpak State University, 742012 Nukus, Uzbekistan
}

\begin{abstract}
We studied the relaxation processes of photoconductivity in $\mathrm{Si}(\mathrm{Li}) p-i-n$ structures. It has been shown that a clearly pronounced "well" is observed in time dependences of the photovoltage pulse after photoexcitation of these structures. Our experimental data are indicative of abnormal relaxation of photoconductivity in silicon $p$ $i-n$ diodes.
\end{abstract}

Keywords: photoconductivity, relaxation, well.

Manuscript received 24.02.10; accepted for publication 08.07.10; published online 30.09.10.

\section{Introduction}

It is known that the most striking example of the inhomogeneous field created by a movable space charge in the semiconductor is the space charge region in $p-n$, $p-i-n$ structures of a large size. Studied in $[1,2]$ was topography of the photo-emf signal associated with the heterogeneity of the electric field in the space charge region in germanium radiation detectors. Silicon $p-i-n$ radiation detectors were studied using the topography of the amplitude spectrum by scanning with a collimated beam of alpha particles [3]. It is important that these studies enabled to reveal significant inhomogeneous distribution of impurities in certain local areas. Study of physical processes in these areas could find unknown physical processes as a base for new fundamental functional principles. Consequently, in semiconductor physics, they can cause a wide interest both of theoreticians and experimentalists $[4,5]$.

\section{Experimental}

In this paper, we consider the relaxation processes for charge carriers in the space charge region inherent to $\mathrm{Si}$ (Li) $p-i-n$ structures. These structures were fabricated by us on the base of wafers made of a $p$-type silicon single crystal with the diameter $50 \mathrm{~mm}$ and thickness $2.5 \mathrm{~mm}$, as well as initial parameters: resistivity $\rho=5000 \mathrm{Ohm} \cdot \mathrm{cm}$, carrier lifetime $\tau=300 \mu \mathrm{s}$. After certain chemical-and-technological operations, lithium diffusion was made on one of plate sides in vacuum at $T=450{ }^{\circ} \mathrm{C}$ down to the depths 320 to $350 \mu \mathrm{m}$. Then, to compensate the whole thickness of the plate, the drift of lithium ions was performed over the entire thickness of it. The drift was carried out in two stages: at $T=80$ $100{ }^{\circ} \mathrm{C}$ and reverse bias voltage 80 to $120 \mathrm{~V}$ and with increasing the latter up to approximately $300 \mathrm{~V}$ at the same temperatures. The end of the drift was fixed by a sharp increase in reverse current through the structure $[6,7]$. The plates prepared using the above-mentioned method were used to measure photoconductivity in their various parts by probing all over the surface. When measuring the photoconductivity, we used LED AL-402 $(\lambda=0.69 \mu \mathrm{m})$ with a radiated power close to $5 \mathrm{~mW}$. Features of the sample photoconductivity were studied using the relaxation curves both for the rise and decay of the photovoltage pulse [8].

\section{Results and discussion}

In separate parts of the investigated samples, the photoconductivity relaxation curves for the decaying photovoltage had a clearly pronounced "well". Fig. 1 shows a typical oscillogram for this type of photoconductivity relaxation. The time is scaled as $1 \mathrm{~ms} / 10 \mathrm{~mm}$ and directed along the abscissa, while the ordinate corresponds to the voltage scaled as $0.05 \mathrm{~V} / 10 \mathrm{~mm}$.

After reaching the maximum photovoltage value $0.3 \mathrm{~V}$, its drop occurs within $1.025 \mathrm{~ms}$, the relaxation curve being of a usual form. Then, in $2.1 \mathrm{~ms}$ one can observe the following sharp drop, and the photovoltage reaches its minimum value equal to $0.05 \mathrm{~V}$. At the beginning of this drop, the photovoltage value was 
$0.12 \mathrm{~V}$. Thus, the slope of the relaxation curve in the interval $1.025-2.1 \mathrm{~ms}$ is $-0.065 \mathrm{~V} / \mathrm{ms}$, while at the beginning of the recession in the interval $0-1.025 \mathrm{~ms}$ the slope of the curve was $-0.175 \mathrm{~V} / \mathrm{ms}$. Consequently, the slope of the second drop is approximately 3 times less than that in the first recession. Between the first and second drops, in the time moment $0.425 \mathrm{~ms}$ the relaxation curve has a minimum slope and can be considered as nearly parallel to the abscissa.

In $2.1 \mathrm{~ms}$ after photoexcitation, the photovoltage is set at the level $0.05 \mathrm{~V}$. In this point of oscillogram, the drop is changed by its growth, i.e., the slope of the curves changes its sign here. Then, in the interval 2.1 to $2.6 \mathrm{~ms}$ the photovoltage increases from $0.05 \mathrm{~V}$ up to $0.075 \mathrm{~V}$, hence, the slope of the curves reaches $0.05 \mathrm{~V} / \mathrm{ms}$. Then again, a slow drop with a slope $0.006 \mathrm{~V} / \mathrm{ms}$ takes place. Thus, the relation curve demonstrates three specific turning points, with one of them where the drop is changed by a growth [3].

It is known that in presence of trapping levels in silicon, the photovoltage pulse value is decreased monotonically during these relaxation processes [9]. Studied in [10] is the influence of the saturation effect for the electron velocity on switching the $n^{+}-p-p^{+}$ structure in the quasi-neutral drift mode. It is noted that the effect of velocity saturation significantly slows down the passage of the Dean wave [11] of electrons through the base of $n^{+}-p-p^{+}$structure, which causes the sharp drop. Proposed in [11] mechanism is rather suitable to explain the appearance of the well in the recession, if we assume that the band gap of silicon clusters contains deep recombination centers [12]. Then, the relaxation time has two components [6]

$\frac{1}{\tau}=\frac{1}{\tau_{0}}+\frac{1}{\tau_{1}}$.

Here, $\tau_{0}$ is the relaxation time inherent to $p$-type semiconductor in the absence of deep recombination centers; $\tau_{1}-$ relaxation time when only these centers are present. Then drop in voltage over time is determined by the formula

$U=U_{s t} e^{-\left(\frac{1}{\tau_{0}}+\frac{1}{\tau_{1}}\right) t}$

Using the experimental results, we determine the time dependence. First of all, let us analyze the function $U$ at the points of extremum, where the first derivative of $U$ with regard to $t$ is equal to zero. In these points, we have

$$
\left(\frac{d \tau_{1}}{d t}\right)_{t_{i}}=\frac{\tau_{1}}{t_{i}}\left(\frac{\tau_{1}}{\tau_{0}}+1\right)
$$

Here, $t_{i}$ is the value of $t$ when one observes a minimum of $U$. The experiment shows that the functional dependence of $U$ on time $t$ has a three-point extremal value. This means that the empirical formula $U$ is a curve of the third power. The equation (3) determines the conditions where the function $U$ has zero derivative. Analyzing the various options for empirical formulas describing the dependence of $U$ on time $t$, we have drawn the conclusion that the following formula is rather convenient for calculations

$-\ln \left(\frac{U}{U_{s t}}\right)=y_{1}+k_{2}\left(\frac{t-t_{k}}{t_{k}}\right)^{2}+k_{3}\left(\frac{t-t_{k}}{t_{k}}\right)^{3}$.

Here, $k_{2}$ and $k_{3}$ depend on the parameters of the function $U$ at the points of extremum:

$k_{2}=y_{0}-y_{1}+k_{3} ; \quad k_{3}=\frac{y_{2}-y_{1}}{a^{2}(a+1)}-\frac{y_{0}-y_{1}}{(a+1)} ;$

$a=\frac{t_{2}-t_{k}}{t_{k}} ; y=\ln \left(\frac{U}{U_{s t}}\right)$.

Then $y_{0}=0$, as in the beginning of recession $U=U_{s t}$. We designate $y_{1}=\ln \left(\frac{U}{U_{s t}}\right)$ at $t=t_{k}$, there $t_{k}$ corresponds to a voltage minimum and $t_{2}$ to maximum $t$. The initial time is determined in a point of recession, therefore $t_{1}=0$. At $t=t_{2}$, one can obtain that $k_{2}$ and $k_{3}$ are defined by the formulas

$$
\begin{aligned}
& k_{2}=\frac{t_{k}^{3} y_{2}}{t_{2}\left(t_{2}-t_{k}\right)^{2}}-\frac{t_{k}+t_{2}}{t_{2}} y_{1}, \\
& k_{3}=\frac{t_{k}^{3}}{t_{2}\left(t_{2}-t_{k}\right)^{2}} y_{2}-\frac{t_{k}}{t_{2}} y_{1} .
\end{aligned}
$$

As

$-\ln \left(\frac{U}{U_{s t}}\right)=t\left(\frac{1}{\tau_{0}}+\frac{1}{\tau_{1}}\right)$,

it follows from (4) that

$t\left(\frac{1}{\tau_{0}}+\frac{1}{\tau_{1}}\right)=y_{1}+k_{2}\left(\frac{t-t_{k}}{t_{k}}\right)^{2}+k_{3}\left(\frac{t-t_{k}}{t_{k}}\right)^{3}$.

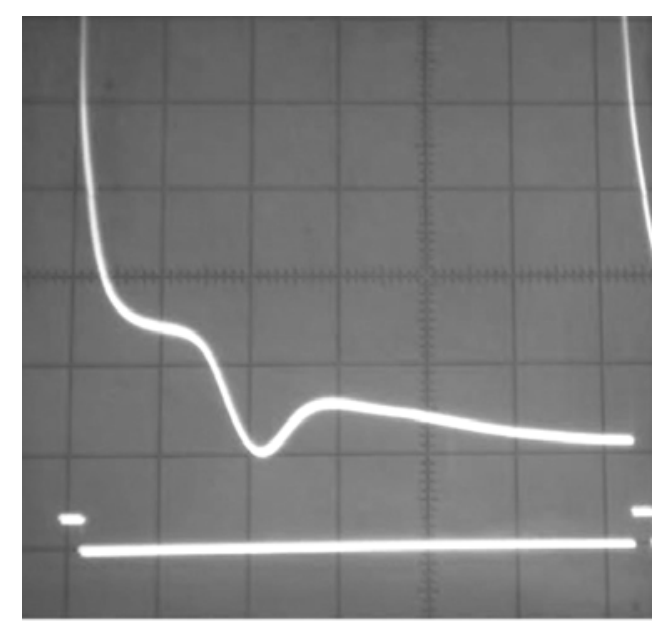

Fig. 1. A relaxation of photoconductivity of $p-i-n$ structures, $y=0.05 \mathrm{~V} / 10 \mathrm{~mm}, x=1 \mathrm{~ms} / 10 \mathrm{~mm}$. 


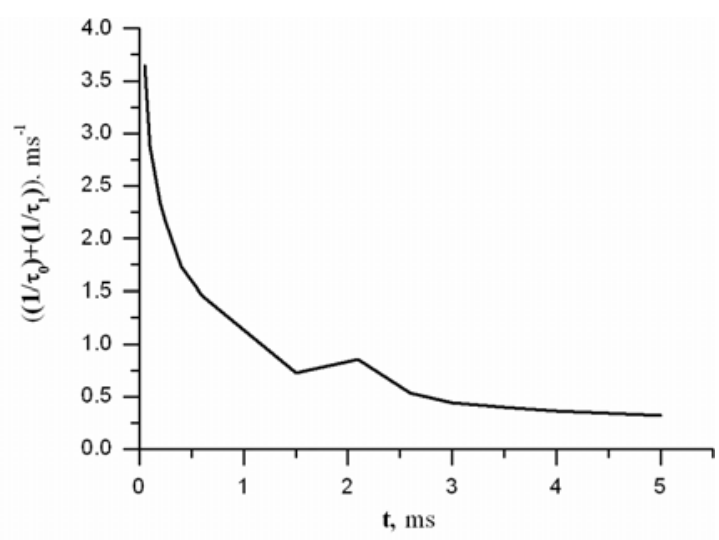

Fig. 2. Graphic dependence of time of a relaxation. Fig. 2.

The graphic plot of $\tau_{1}$ versus time is shown in

As seen, in the process of approach to the abscissa the pulse photovoltage value takes its minimum, time of relaxation starts to decrease, when the speed of reduction in $\tau_{1}$ value with time up to the certain value determined using the formula (3) reaches its minimal value. Here, $\tau_{1}$ begins to increase up to a certain maximum, after that one can observe a quasi-stationary value of the relaxation time. As it was noted above, the account of trapping levels does not give extreme points in the range of recession in the pulse photovoltage value [9]. More exact calculation of dynamic characteristics $n^{+}-p-p^{+}$structures, by means of Dean waves for electrons gives monotonic recession of a photovoltage on time, too [10]. However, to ascertain the specific nature of defects causing this non-monotonic drop in conductivity inherent to $p-i-n$ diodes, additional investigations are necessary.

\section{Conclusions}

Thus, our experimental data are indicative of abnormal relaxation of photoconductivity in silicon $p-i-n$ diodes.

\section{References}

1. N.B. Strocan, N.I. Tisnek, V.F. Afanasev // Fizika tekhnika poluprovodnikov 5, p. 211 (1968), in Russian.

2. V.F. Afanasev, N.B. Strocan, N.I. Tisnek // Fizika tekhnika poluprovodnikov 2, p. 66 (1970), in Russian.

3. S.A. Azimov, R.A. Muminov, S.H. Shamirzaev, A.J. Jafasov // Silicon-Lithium Detectors of Nuclear Radiation. FAN, Tashkent, 1981 (in Russian).

4. S.M. Sze, Physics of Semiconductor Devices. Wiley-Intersci. Publ., Toronto, Singapore, 1981.

5. K.F. Brennan, Physics of Semiconductors with Application to Optoelectronic Devices. Cambridge University Press, 1999.

6. S.A. Radjapov // Uzbek Journal of Physics 9(3), p. 190-194 (2007).

7. S.A. Radjapov // DAS RUz, No. 2, p. 17-21 (2007).

8. S.M. Ryvkin, Photo-electric Phenomena in Semiconductors. Phys. Math. Lit. Publ., Moscow, 1963 (in Russian).

9. D.A. Aronov, V. Zaytova, Photomagnetic Effect and Photoconductivity in Semiconductors at High Levels of Excitation. FAN, Tashkent, 1987 (in Russian).

10. T.T. Mnatsakanov, M.E. Levinshtein, A.G. Tandoev, and S.N. Yurkov, Specific features of dynamic injection and base layer modulation processes in power $n^{+}-p-p^{+}$diodes // Semiconductors 41, No. 11, p. 1381-1387 (2007).

11. R.H. Dean // J. Appl. Phys. 46, p. 585 (1969).

12. S.Zh. Karazhanov, É.V. Kanaki, Electrical properties of semiconductors with pair defects // Semiconductors 34, No. 8, p. 880-885 (2000). 